\title{
An exploratory study of the implementation of early intervention workshops for primary caregivers in Johannesburg
}

\author{
S Medhurst, ${ }^{1}$ BA (Speech \& Hearing Therapy); S Abdoola, ${ }^{2}$ BComm Pathology (SLT \& A), MECI; \\ L Duncan, ${ }^{3}$ BSocSci (Psych), BEd Hon (School Guidance and Counselling), MECI \\ ${ }^{1}$ Speech-Language Therapist and Audiologist, Johannesburg, South Africa \\ ${ }^{2}$ Department of Speech Pathology and Audiology, Faculty of Humanities, University of the Witwatersrand, Johannesburg, South Africa; and \\ Department of Speech Language Pathology and Audiology, Faculty of Humanities, University of Pretoria, South Africa \\ ${ }^{3}$ Remedial Therapist, Reddford House, Pretoria, South Africa
}

Corresponding author: S Abdoola (786.shabnam@gmail.com)

\begin{abstract}
Background. If primary caregivers are able to stimulate their children's development effectively, then the prevalence of children at risk of cognitive and language developmental delays could decrease and the shortage of available services for the identified children could be addressed, as hopefully fewer children would require extensive early intervention (EI) services later on in life.

Objective. To develop and implement an EI workshop with primary caregivers on how to provide language and cognitive stimulation through daily living activities (DLAs).

Methods. Two workshops were conducted at two daycare centres, focusing on daily language stimulation, with the primary caregivers of children aged between 0 and 3 years. A pre-workshop, semi-structured group interview was conducted to gain insight into the participants' knowledge and expectations. This was followed with a post-workshop, semi-structured group interview to gain insight into and feedback on how the participants were able to carry over the techniques in order to stimulate their children in DLAs. The data were analysed using thematic data analysis.

Results. It was found that although participants demonstrated the basic understanding of the concept of EI, their knowledge improved with the workshop. The participants reported that they were able to implement the techniques gained from the workshop and noticed a change in their children's behaviour and communication within the space of 1 week. The participants also reported on the ease of stimulating their children through DLAs and that no additional time had to be scheduled for stimulation.

Conclusion. The workshops have the potential to target populations regardless of their socioeconomic status, cultural beliefs, linguistic differences, and access to medical institutions.
\end{abstract}

S Afr J Child Health 2016;10(1):71-74. DOI:10.7106/SAJCH.2016.v10i1.1057

The literature indicates a direct correlation between poor academic performance (failure to either complete primary or secondary school, or failure to obtain the requirements to complete each grade of schooling) in primary caregivers (main parent or family member who takes care of and spends most time with the child) and poor cognitive and language development in the children of these primary caregivers. Children from low-income households generally experience less consistent caregiving, support and cognitively stimulating home environments, alongside external environment influences. ${ }^{[1]}$ It is therefore essential to enrich these children's home environments in order to support cognitive development ${ }^{[2]}$ and, consequently, language development.

Owing to the fact that there is a lack of speech-language pathologists in the lower socioeconomic status areas in South Africa (SA), it becomes necessary to provide informative workshops to these families. The objective of the workshops provided in the current study was to provide techniques to primary caregivers on how to stimulate cognitive and language development of their children in daily living activities (DLAs). The use of these techniques by the primary caregivers might decrease the need for future intervention for these children at risk from cognitive and language developmental delays.

\section{Early intervention (EI)}

EI refers to comprehensive activities designed to improve and develop the cognitive, language, motor and sensory development of young children. ${ }^{[3,4]}$ Three major objectives of EI are to identify at-risk children, to provide EI as a preventive measure, and to identify and intervene with young children that have existing developmental delays. ${ }^{[3]}$ EI services can be implemented regardless of the socioeconomic status of the participants. ${ }^{[5]}$ The evidence supports and indicates that EI has positive effects on development if implemented correctly by the therapists in partnership with the parents or caregivers. ${ }^{[5]}$ However, there appears to be insufficient literature discussing how primary caregivers can be empowered to stimulate their children optimally in order to prevent developmental delays and to address the lack of intervention services in the public health services in SA. Family interaction influences the cognitive and language development of young children. ${ }^{[6]}$ Therefore, it is crucial to involve primary caregivers in EI services and consequently empower them.

\section{Caregivers and EI services}

Caregivers are the most critical providers of care, support, holistic development and stimulation, and therefore play a vital role in EI. ${ }^{[7]}$ Furthermore, open and honest communication between all parties is essential for the implementation of EI. It is also vital that caregivers understand the different stages of development and how to stimulate their child's development. An audit conducted by the SA Department of Education in 2000 revealed that $84 \%$ of young children between the ages of 0 and 5 years of age did not have access to formal early childhood development (ECD) services and relied on their primary caregivers for EI ${ }^{[8]}$ Children from low-income socioeconomic environments face external factors that affect their development, ${ }^{[1]}$ which further stresses the importance for primary caregivers to have access to EI services and implement the services. 
The purpose of this qualitative study was to develop and implement an EI workshop for primary caregivers on how to provide language and cognitive stimulation. The caregivers were required to implement the shown skills at home in DLAs and provide feedback as to whether or not it was challenging to stimulate their child's language and cognition, and if any changes in their child's behaviour, cognition or language were noted within a week. As previously mentioned, there is a lack of speech-language pathologists in SA and providing workshops in a group setting is more effective than locating individual caregivers and providing information.

\section{Methods \\ Participants}

A combination of convenience and snowball sampling was used in selecting the participants from two daycare centres in Johannesburg. The daycare centres had to each be registered as a child development centre. The daycare centres were contacted and asked if any EI services were being offered to parents and if not, would they be interested in receiving EI services? A needs analysis was conducted at the two selected daycare centres to further specify the needs in terms of EI services. The participants had to be caregivers of children between the ages of 0 and 3 years; this is the age group stipulated as the target group for EI in SA. ${ }^{[4]}$ The sample size comprised 15 participants, combined of primary caregivers and staff members from the daycare centres.

\section{Data collection}

Data were collected through semi-structured group interviews with the participants. Semi-structured group interviews are more flexible than standardised questionnaires but are not unstructured discussions, and they encourage participants to respond to openended questions; this in turn reveals the participants' knowledge, opinions and concerns regarding the topics being discussed. ${ }^{[9]}$ Semi-structured group interviews are seen to have high validity because the participants are able to discuss items in great detail and depth. ${ }^{[10]}$ There were two group interviews: a pre-workshop interview to determine the primary caregivers' general understanding and thoughts around EI and their expectations of the workshops, and a post-workshop interview to receive the primary caregivers' feedback from implementing and carrying over the techniques shown into the DLAs at home. The pre-workshop interview was conducted on the same day as the workshop. The post-workshop interview was conducted 1 week later in order to allow the participants to implement the knowledge and skills acquired from the workshop. The time period between the data collection from Daycare Centre 1 and Daycare Centre 2 was $\sim 2$ weeks. The information obtained from the interviews was recorded with an audio recorder.

\section{Workshops}

The workshops were conducted and facilitated by the same student speech-language therapist. Although there are few standardised EI programmes available, the workshop was based on the principles of Guralnick's Developmental Systems Approach (DSA). The workshop provided information pertaining to EI, and importance of EI services, age-appropriate communication and cognitive developmental milestones, and general motor developmental milestones. It is essential for the primary caregivers to have a general understanding of the holistic development of their children, and when and where to seek help if their children are not developing at age-appropriate levels. The primary objective of the workshop was to look at how primary caregivers can stimulate children through DLAs, including feeding, bathing and dressing, in order to foster the carryover of skills. ${ }^{[1]]}$

\section{Reliability and validity}

Reliability was ensured by conducting the same methodology at the two different daycare centres, and utilising a field journal or researcher's 'reflective commentary' to limit researcher bias and influence of the researcher's emotions when analysing the data. Validity was ensured through triangulation, specifically of data sources, to verify the viewpoints and experiences of the participants against each other's. ${ }^{[12]}$ A variety of participants (primary caregivers of children between the ages of 0 and 3 years, and daycare staff members) were included in the sample population.

\section{Ethical considerations}

Ethical clearance for this study was obtained from the Human Research Ethics Committee (Non-Medical) of the University of the Witwatersrand (protocol number: H14/02/04).

Confidentiality and anonymity could not be guaranteed because of the workshops and semi-structured group interviews, which were discussed with the participants. However, personal details and names of participants have been omitted in the presentation of findings to ensure confidentiality and anonymity.

\section{Results \\ Workshops}

There were 12 participants from Daycare Centre 1. Of these, three were male and nine were female. Nine participants were primary caregivers and three were staff members from that daycare centre. There were 10 participants from Daycare Centre 2. Of these, one was male and nine were female. Six participants were primary caregivers and four were staff members (one from that daycare centre and three from neighbouring centres). Both Daycare Centres 1 and 2 had one child receiving EI services such as physiotherapy or speech and language therapy. No other EI services were being provided at either Daycare Centre 1 or 2.

Thematic content analysis revealed five main themes.

\section{Awareness of EI}

The participants from both Daycare Centres 1 and 2 had limited awareness and understanding of the term EI. One participant stated: 'I've heard of EI on programmes, children's programmes, but I'm not clear of its meaning. Another participant demonstrated a basic understanding of the term: 'You intervene if you see there's a problem or before the problem starts', but could not identify the age group at which EI is targeted. The primary caregivers demonstrated an understanding of how their child's current development affects school readiness: 'ECD is very much important. If you can go wrong there, then you mess up big time. I think most of the parents understand, but I think it's also ignorance, but there were a few participants who stated that their child is not talking now but they will wait until they are around 4 - 5 years old before asking for help.

\section{Access to information}

The three main areas from which participants receive information are clinics, daycare centres and television/technology. The majority of the participants from Daycare Centres 1 and 2 stated that they received information pertaining to their child's development from the nurses or doctors at the clinics, especially when the child goes for immunisation. The daycare centres are also responsible for reporting to caregivers about their child's development. Information is readily available through the internet, and participants admitted to using the internet to gather information about their own children and about conditions or syndromes that other children present with at the daycare centre or that they have heard of.

\section{Current beliefs}

When the topic of cultural belief was addressed during the preworkshop interviews, all the participants agreed on seeking medical intervention instead of consulting traditional healers. When probed further, some of the participants stated that religion is a major 
influence in the change in belief, as many community members are becoming Christian/'born-again Christian, which influences the way they view medical intervention versus traditional intervention. It was also mentioned that education and access to information through technology also influences the participants' beliefs.

\section{Obtaining information}

All the participants stated that they attended the workshops to receive information about development and how to stimulate their child's development. Most participants from Daycare Centre 1 wanted information with regard to tantrums, and participants from both Daycare Centres 1 and 2 expressed specific concerns about their children's communication skills or behaviour.

\section{Daycare centre feedback \\ Daycare Centre 1}

The participants showed a slight increase in understanding of the term EI: 'I think I can say it's how the child develops and how to interact with the child'; '...you can also identify a phase where the child needs help and work on that.' The participants provided examples of how they stimulated their children during that week and the outcomes of the stimulation: 'You know I even tried to make her to sit down and eat by herself at least. It worked, and I really can see that she knows what she is doing and she is listening. She was well behaved this week, but I can see that before workshop she was just toying with me'; 'Just to speak to my child, what do you want to wear, this colour or this colour? I know what colours he likes now'; 'To deal with the tantrums - before he would cry for nothing and now I can control them. He is better and I can give him options to choose what he wants and there is a change now'; 'I think I was able to involve him in preparing the food, he likes that and that changed his eating a lot, because he was very fussy.' The participants also stated that they spoke to other parents at the daycare centre about the workshops, and shared information that they learnt from the workshop.

\section{Daycare Centre 2}

Although the post-workshop could not be conducted, the email received about feedback stated: 'I really gained a lot from the workshop and everyone else that was in the workshop. I think if it wasn't for the other commitments that they had, they would've attended again, because I received good feedback from them. They told me that they've been practising the things that you told us to practise, like talking to your child like you are talking to an adult and that every time that you get to spend time with your child make it a learning opportunity'

\section{Discussion}

The awareness of EI is important, because if caregivers are not aware that these services are available, then preventive and curative management for at-risk children or children who require the services cannot be and are not being implemented. ${ }^{[3]}$ Caregivers' knowledge and thoughts regarding EI are affected by the means in which they gather information about child development. Cultural beliefs, especially towards medical intervention, influence whom caregivers seek information from pertaining to a child's development, as well as whether or not they receive or continue with EI services. Grandparents and parents might also have diverse beliefs regarding interventions, which can be obstacles when young children are in need of EI.

Although only two workshops were conducted, the feedback from the workshops provided insight into the potential and the importance of holding such workshops. There was good feedback from all participants, as they were able to implement the strategies given to them into their DLAs, and they noted changes in their children's behaviour and became more aware of their children's abilities over the course of 1 week. It would have been more beneficial to monitor this over a longer period of time, as more insight into the effectiveness of the workshops could have been gained. It is vital that primary caregivers are aware of how to stimulate a child's cognitive and language skills in order to foster age-appropriate development. If a child is not developing age appropriately, the primary caregivers need to be able to identify this, be aware that EI services are available and know how such services can assist with the improvement of the child's development. Primary caregivers also need to be made aware of how a child's current development will affect school readiness and academic performance. The workshop covered all these areas, provided information on how to stimulate the children's language and cognition functionally through DLAs, and covered relevant areas that the participants could relate to. There was a slight increase in understanding of the concept and benefits of the term 'EI' after the workshop.

Participants stated that the places from which they access information regarding children's development include clinics, daycare centres and television or internet. Various allied professionals could provide information at clinics through pamphlets and posters, and possibly implement workshops or support groups at the clinics, but this would have to be discussed with therapists at the clinics and the Department of Health. This study indicated that direct preventive and curative intervention can occur at the daycare centres. Cultural beliefs did not seem to have an effect on medical intervention; change in beliefs seems to be due to religion and primary caregivers having more access to information. This finding was common among participants at both daycare centres.

This study focused on the primary caregivers using the techniques shown to them through the implementation of EI workshops to provide language and cognitive stimulation through DLAs. However, the involvement of the daycare centres became apparent, including the importance of their roles within the community and in terms of sharing the information gained during the workshops. The daycare centres are now responsible for facilitating the transfer of the workshop information to primary caregivers who were unable to participate in the study, and the participants can share knowledge with other caregivers who did not participate in the study. Although it was not an objective of the study, Daycare Centre 1 held a workshop for the other caregivers who were unable to participate in the original workshop. This workshop was based on the workshop presented during the study. This provides hope for the extension of workshops should these be implemented in more daycare centres in the future.

\section{Study limitations}

It was difficult to identify and compare themes as the post-workshop interview could not be conducted at Daycare Centre 2. However, the owner of Daycare Centre 2 emailed feedback. If this study were to be conducted in future, more daycare centres should be involved and the study should be conducted over a longer time period to better measure the effectiveness of the workshops.

\section{Recommendations}

In order to measure the effectiveness of the workshops, it would be essential to implement the workshops at more daycare centres, and conduct sufficient follow-up interviews. The workshops can also be implemented in areas with different socioeconomic statuses and with varying access to health facilities where EI services are available, to determine how the workshops can target different populations.

\section{Conclusion}

Although only two workshops and one post-workshop interview were conducted, this study provided valuable insight into the importance of providing EI workshops to primary caregivers. The study also indicated that primary caregivers are able to stimulate their children's 
communication and cognition more easily through DLAs than scheduling additional time in the evening or on weekends to provide the stimulation. There is potential for conducting workshops that can incorporate different health and allied professionals and which can target populations regardless of their socioeconomic status, cultural beliefs, linguistic differences, and access to medical facilities.

\section{References}

1. Votruba-Drzal E, Coley RL, Chase-Lindsdale PL. Child care and low-income children's development: Direct and moderated effects. Child Dev 2004;75(1):296312. [http://dx.doi.org/10.1111/j.1467-8624.2004.00670.x]

2. Woolfolk A. Educational Psychology (10th ed.). Boston, USA: Pearson Education, Inc., 2007.

3. Shonkoff JP, Meisels SJ. Handbook of Early Childhood Intervention (2nd ed.) Cambridge: Cambridge University Press, 2000.

4. Department of Social Development. Guidelines for Early Childhood Development Services. Pretoria, South Africa: United Nations Children's Fund, 2006.
5. Guralnick MJ. Effectiveness of early intervention for vulnerable children: A developmental perspective. Am J Ment Retard 1998;102(4):319-345.

6. Guralnick MJ. Family influences in early development: Integrating the science of normative development, risk and disability and intervention. In: McCartney K, Phillips D (eds). Handbook of Early Childhood Development. Oxford; Blackwell Publishers, 2006:44-61.

7. UNICEF. Guidelines for Early Childhood Development Services. Pretoria, South Africa: Department of Social Development, 2006.

8. UNICEF. Young Lives: Statistical Data on the Status of Children Aged 0 - 4 in South Africa. Pretoria, South Africa: United Nations Children's Fund, 2007.

9. Lodico MG, Spaulding DT, Voegtle KH. Methods in Educational Research: From Theory to Practice. (2nd ed.). San Francisco: Wiley \& Sons, Inc., 2010.

10. Dixit JB, Kumar R. Structured System Analysis and Design. New Delhi: Laxmi Publications, 2007.

11. Sussman F. The Power of Using Everyday Routines to Promote Young Children's Language and Social Skills. Toronto: Hanen Early Program Centre, 2014.

12. Shenton AK. Strategies for ensuring trustworthiness in qualitative research projects. Educ Inf 2004;22:63-75. 